\title{
Crystal structure of tetracerium(III) trisulfide heptaoxodisilicate(IV), $\mathrm{Ce}_{4} \mathrm{~S}_{3}\left[\mathrm{Si}_{2} \mathrm{O}_{7}\right]$
}

\author{
I. Hartenbach and Th. Schleid* \\ Universität Stuttgart, Institut für Anorganische Chemie, Pfaffenwaldring 55, D-70569 Stuttgart, Germany
}

Received February 26, 2002, accepted and available on-line May 7, 2002; CSD-No. 409606

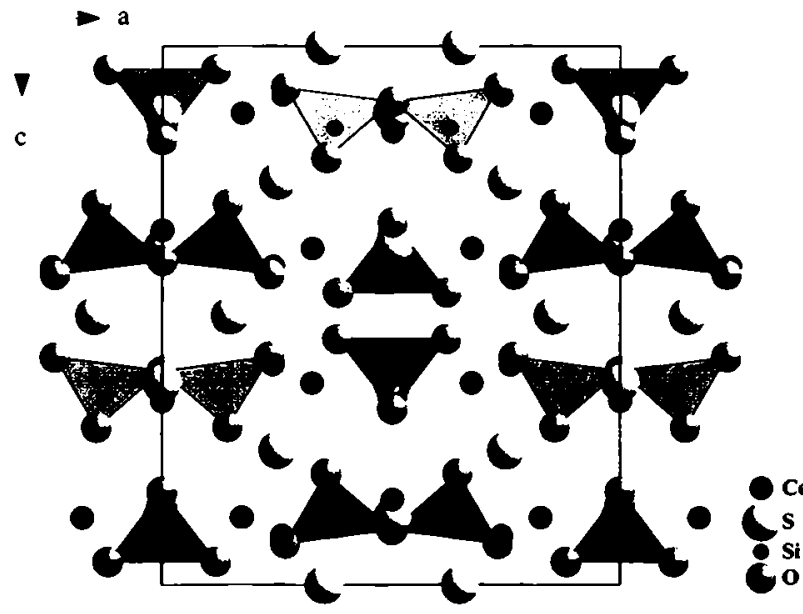

Abstract

$\mathrm{Ce}_{4} \mathrm{O}_{7} \mathrm{~S}_{3} \mathrm{Si}_{2}$, tetragonal, $I 41$ /amd (No. 141), $a=12.0543(8) \AA$, $c=14.2351(9) \AA, V=2068.4 \AA^{3}, Z=8, R_{\mathrm{gt}}(F)=0.027$, $w R_{\text {ref }}\left(F^{2}\right)=0.060, T=298 \mathrm{~K}$.

\section{Source of material}

Colourless transparent single crystals of $\mathrm{Ce}_{4} \mathrm{~S}_{3}\left[\mathrm{Si}_{2} \mathrm{O}_{7}\right]$ were obtained as almost regular octahedra by the reaction of cerium dioxide and cerium with sulfur and silicon dioxide (molar ratio 3:1:6:4) in an excess of molten cerium trichloride as fluxing agent at $1123 \mathrm{~K}$ for seven days in an evacuated silica ampoule. The crystals of the title compound emerged as main product (along with traces of $\mathrm{CeOCl}$ and $\mathrm{Ce}_{2} \mathrm{O}_{2} \mathrm{~S}$ as by-products) and remain stable to air and water.

\section{Discussion}

Although compounds of the general formula $\mathrm{M}_{4} \mathrm{~S}_{3}\left[\mathrm{Si}_{2} \mathrm{O}_{7}\right](\mathrm{M}=$ $\mathrm{La}-\mathrm{Nd}, \mathrm{Sm}, \mathrm{Gd}-\mathrm{Yb}$ ) are already known [1-8], it was not possible to synthesize the single-crystalline isotypic cerium compound up to now [9].

The title compound contains isolated pyroanionic $\left[\mathrm{Si}_{2} \mathrm{O}_{7}\right]^{6-}$ units surrounded by twelve cerium atoms as terminal and edgespanning ligands. The $(\mathrm{Ce} 1)^{3+}$ cations are even able to connect the bridging oxygen $(\mathrm{O} 3)$ although this $\mathrm{Ce}-\mathrm{O}$ distance is rather long (326 pm). Both crystallographic independent $\mathrm{Ce}^{3+}$ cations show tricapped trigonal prisms $(\mathrm{CN}=9)$ as coordination polyhedra built of four oxygen and five sulfur atoms in case of $\mathrm{Cel}$ but seven $\mathrm{O}^{2-}$ and two $\mathrm{S}^{2-}$ for $\mathrm{Ce} 2$. The $\mathrm{Ce}^{3+}-\mathrm{O}^{2-}$ distances with values of $241 \mathrm{pm}-267 \mathrm{pm}$ (except the very long distance of 326 pm mentioned before) range in the region expected for those separations just like the $\mathrm{Ce}^{3+}-S^{2-}$ bond lengths $(d(\mathrm{Ce}-S)=290 \mathrm{pm}$ $-344 \mathrm{pm}$ ). All oxygen atoms belong to vertex-shared double tetrahedra of disilicate anions $\left[\mathrm{Si}_{2} \mathrm{O}_{7}\right]^{6-}$ showing $\mathrm{Si}-\mathrm{O}$ distances of $161 \mathrm{pm}-164 \mathrm{pm}$ and $\mathrm{O}-\mathrm{Si}-\mathrm{O}$ angles of $104^{\circ}-120^{\circ}$ very much like almost every other compound containing single or vertex-connected $\left[\mathrm{SiO}_{4}\right]^{4-}$ tetrahedra. The bridging angle ( $\mathrm{Si}-\mathrm{O}-\mathrm{Si}$ ) of the disilicate unit lies with $132^{\circ}$ close to those of the A- and G-type rare-earth disilicates $\left(\mathrm{M}_{2}\left[\mathrm{Si}_{2} \mathrm{O}\right.\right.$ ] $]$ ) [10]. Finally, the crystal structure distinguishes three $\mathrm{S}^{2-}$ anions that once exhibit an environment of six $\mathrm{Ce}^{3+}$ cations in the shape of a rather distorted octahedron ( $\mathrm{S} 1$ ), and twice one of four cerium atoms either forming a seesaw (S2) or a flat square (S3), respectively. The whole structure consists of layers of disilicate anions parallel (001) containing both types of cerium cations as well as the (S2) ${ }^{2-}$ and the (S3) ${ }^{2-}$ anions. These are separated by the high-coordinated sulfide atoms in position $\mathrm{S} 1$. The stacking order can be considered as A, -B, B, - A where A and B are the same layers but perpendicular rotated against each other around the $c$-axis, as can be seen in the figure.

Table 1. Data collection and handling.

$\begin{array}{ll}\text { Crystal: } & \begin{array}{l}\text { colourless octahedron, } \\ \text { size } 0.08 \times 0.08 \times 0.08 \mathrm{~mm}\end{array} \\ \text { Wavelength: } & \text { Mo } K_{\alpha} \text { radiation }(0.71069 \AA) \\ \mu: & 181.17 \mathrm{~cm}^{-1} \\ \text { Diffractometer, scan mode: } & \text { Nonius Kappa-CCD, } \varphi / \omega \\ 2 \theta_{\max }: & 55.3^{\circ} \\ N(h k l)_{\text {measured, }} N(h k l)_{\text {hnique: }} & 9174,669 \\ \text { Criterion for } I_{\text {obs }}, N(h k l)_{\text {gt: }}: & I_{\text {obs }}>2 \sigma\left(I_{\text {obs }}\right), 616 \\ N(\text { param })_{\text {hefined: }} & 47 \\ \text { Program: } & \text { SHELXL-97 }[11]\end{array}$


Table 2. Atomic coordinates and displacement parameters (in $\AA^{2}$ ).

\begin{tabular}{|c|c|c|c|c|c|c|c|c|c|c|}
\hline Atom & Site & $x$ & $y$ & $z$ & $U_{11}$ & $U_{22}$ & $U_{33}$ & $U_{12}$ & $U_{13}$ & $U_{23}$ \\
\hline $\mathrm{Ce}(1)$ & $16 h$ & 0 & $0.01366(4)$ & $0.34060(4)$ & $0.0154(3)$ & $0.0176(3)$ & $0.0155(3)$ & 0 & 0 & $-0.0014(2)$ \\
\hline $\mathrm{Ce}(2)$ & $16 g$ & $0.17510(3)$ & $x+1 / 4$ & $7 / 8$ & $0.0100(2)$ & $U_{11}$ & $0.0112(3)$ & $0.0011(2)$ & $-0.0001(1)$ & $-U_{13}$ \\
\hline$S(1)$ & $16 f$ & $0.3512(2)$ & 0 & 0 & $0.0066(9)$ & $0.027(1)$ & $0.010(1)$ & 0 & 0 & $0.0072(9)$ \\
\hline$S(2)$ & $4 b$ & 0 & $1 / 4$ & $3 / 8$ & $0.009(1)$ & $U_{11}$ & $0.016(2)$ & 0 & 0 & 0 \\
\hline$S(3)$ & $4 a$ & 0 & $3 / 4$ & $1 / 8$ & $0.005(1)$ & $U_{11}$ & $0.013(2)$ & 0 & 0 & 0 \\
\hline $\mathrm{Si}$ & $16 h$ & 0 & $0.3728(2)$ & $0.0959(2)$ & $0.0030(9)$ & $0.0048(9)$ & $0.008(1)$ & 0 & 0 & $-0.0004(8)$ \\
\hline$O(2)$ & $16 h$ & 0 & $0.0250(4)$ & $0.1715(4)$ & $0.010(3)$ & $0.005(2)$ & $0.009(3)$ & 0 & 0 & $0.004(2)$ \\
\hline$O(3)$ & $8 e$ & 0 & $1 / 4$ & $0.6074(5)$ & $0.010(4)$ & $0.019(4)$ & $0.005(4)$ & 0 & 0 & 0 \\
\hline
\end{tabular}

Acknowledgments. The authors gratefully acknowledge the support of the Deutsche Forschungsgemeinschaft (Bonn, Germany), the Fonds der Chemischen Industrie (Frankfurt/Main, Germany) and the State of Baden-Württemberg (Stuttgart, Germany).

\section{References}

1. Siegrist, T.; Petter, W.; Hulliger F.: Samarium Pyrosilicate Sulfide, $\mathrm{Sm}_{4} \mathrm{~S}_{3}\left[\mathrm{Si}_{2} \mathrm{O}_{7}\right.$ ]. Acta Crystallogr. B38 (1982) 2872-2874.

2. Grupe, M.; Lissner, F.; Schleid, Th.; Urland, W.: Chalkogenid-Disilicate der Lanthanoide von $\mathrm{Typ}_{4} \mathrm{M}_{3}\left[\mathrm{Si}_{2} \mathrm{O}\right](\mathrm{M}=\mathrm{Ce}-\mathrm{Er}, \mathrm{X}=\mathrm{S}$, Se). Z. Anorg. Allg. Chem. 616 (1992) 53-60.

3. Range, K.-J.; Andratschke, M.; Gietl, A.: Crysal structure of ytterbium(III) trisulfide disilicate $\mathrm{Yb}_{4} \mathrm{~S}_{3}\left[\mathrm{Si}_{2} \mathrm{O}\right]$ ]. Z. Kristallogr. NCS 211 (1996) 816.

4. Sieke, C.: Der Einfluss von Sulfid- und Chloridanionen auf die Kristallchemie von Silicaten, Boraten und Phosphaten dreiwertiger Lanthanide. Dissertation, University of Stuttgart, Germany 1998.

5. Sieke, C.; Schleid, Th.: $\mathrm{Sm}_{4} \mathrm{~S}_{3}\left[\mathrm{Si}_{2} \mathrm{O}_{7}\right]$ und $\mathrm{NaSm}_{9} \mathrm{~S}_{2}\left[\mathrm{SiO}_{4}\right] 6: \mathrm{Zwei}$ Sulfidsilicate mit dreiwertigem Samarium. Z. Anorg. Allg. Chem. 625 (1999) 131-136.

6. Zeng, H.-J.; Mao, J.-G.; Huang, J.-Sh.: Synthesis and crystal structure of $\mathrm{La}_{4} \mathrm{~S}_{3}\left[\mathrm{Si}_{2} \mathrm{O}\right]$ ]. J. Alloys Compds. 291 (1999) 89-93.
7. Sieke, C.; Schleid, Th.: $\mathrm{Pr}_{4} \mathrm{~S}_{3}\left[\mathrm{Si}_{2} \mathrm{O}_{7}\right]$ und $\mathrm{Pr}_{3} \mathrm{Cl}_{3}\left[\mathrm{Si}_{2} \mathrm{O}\right]$ ]: Durch weiche Fremdanionen modifizierte Derivate von Praseodymdisilicat. $Z$. Anorg. Allg. Chem. 626 (2000) 196-201.

8. Hartenbach, I.; Sieke, C.; Schleid, Th.: $\mathrm{M}_{4} \mathrm{~S}_{3}\left[\mathrm{Si}_{2} \mathrm{O}_{7}\right](\mathrm{M}=\mathrm{Gd}-\mathrm{Yb})$ : Sulfid-Oxodisilicate der schweren Lanthanoide. Z. Naturforsch. 57b (2002), to be submitted.

9. Hartenbach, I.: Anionenderivatisierte Oxo- und kationenderivatisierte Thiosilicate der Selten-Erd-Elemente. Dissertation, University of Stuttgart, Germany 2001, Mensch \& Buch Verlag Berlin, ISBN 3-89820-294-1.

10. Felsche, J.: The Crystal Chemistry of Rare-Earth Silicates. In: Structure and Bonding 13, p. 99-197, Springer-Verlag (Berlin, Heidelberg, New York) 1973.

11. Sheldrick, G. M.: SHEL XL-97. Program for refining crystal structures. University of Göttingen, Germany 1997. 